OPEN ACCESS

Edited by:

Masoud Mozafari

University of Toronto, Canada

Reviewed by:

Volker Martin Lauschke,

Karolinska Institutet (KI), Sweden

Neil Lagali,

Linköping University, Sweden

*Correspondence:

Ovidiu Samoila

iovidius@yahoo.com

Specialty section:

This article was submitted to Gene and Cell Therapy,

a section of the journal

Frontiers in Medicine

Received: 17 June 2019

Accepted: 29 January 2020

Published: 18 February 2020

Citation:

Samoila O and Gocan D (2020)

Clinical Outcomes From Cultivated Allogenic Stem Cells vs. Oral Mucosa Epithelial Transplants in Total Bilateral

Stem Cells Deficiency

Front. Med. 7:43

doi: 10.3389/fmed.2020.00043

\section{Clinical Outcomes From Cultivated Allogenic Stem Cells vs. Oral Mucosa Epithelial Transplants in Total Bilateral Stem Cells Deficiency}

\author{
Ovidiu Samoila* and Diana Gocan \\ Department of Ophthalmology, "Iuliu Haţieganu" University of Medicine and Pharmacy, Cluj-Napoca, Romania
}

Total bilateral limbal stem cell deficiency results from various pathologies, from burns (either chemical or physical) to Sjogren Syndrome, aniridia or ocular cicatricial pemphigoid. After the loss of stem cells, normal corneal epithelium is replaced by a more opaque and vascularized conjunctival epithelium, causing loss of vision. After 1997, cultivation techniques for limbal stem cells became possible. In parallel, cultivation techniques for oral mucosa epithelial cells were also available. The aim of our review was to summarize the clinical outcomes following allogenic cultured limbal stem cell transplant (allogenic CLET), and on the other hand, oral mucosa derived epithelium transplant (cultivated oral mucosa epithelial transplant-COMET or cultivated autologous oral mucosal epithelial cell sheet-CAOMECS), in the case of total bilateral limbal stem cell loss. Thirty studies matching the inclusion criteria were found. The clinical improvement in both methods was reported similar, with percentages higher than $50 \%$ of the treated cases. However, the comparison between studies was difficult to achieve due to the lack of a universal and objective grading tool for assessing post-operative results. The definition of clinical improvement was problematic, because success was defined differently, depending on the study. Moreover, some of the studies followed both autologous and allogenic CLET, but described the results together, for both procedures, and therefore it was impossible to analyze them separately. COMET presented some advantages compared to CLET. By using autologous cells, there was no risk of immune activation and no immunosuppression was needed. COMET, however, might be associated with increased risk of persistent epithelial defects and graft failure, compared with allogenic CLET.

Keywords: stem cells, cornea, cultivation techniques, allogenic, limbal transplant, oral mucosa

\section{INTRODUCTION}

The reservoir of corneal epithelial stem cells, a quiescent cell population with proliferative capacity, is located in a niche in the palisades of Vogt, deep in the structure of the limbus, in the basal epithelial layer. Their distribution along the limbus is not regular; they are mostly present in the nasal region and in the mid or distal portion of the limbus toward the conjunctiva. In addition to stem cells, niches consist also of non-stem cells, such as limbal stromal fibroblasts, melanocytes and immune cells which have the role of maintaining the stem cells dormant $(1,2)$. Following an 
injury of the corneal surface, the limbal epithelial stem cells (LESCs) proliferate, divide, migrate and mature in order to ensure regeneration (3). LESCs are activated into transient amplifying cells, divide and migrate toward the center of the cornea in order to restore the surface. LESC activation is dependent on growth factors, cytokines, extracellular matrix, and integrin receptors $(2,4)$. Dysfunctional or destroyed LESCs define corneal limbal stem cell deficiency (LSCD). Clinically, LSCD causes loss of corneal transparency and replacement of the corneal epithelium with conjunctival epithelium, scarring, vascularization, and persistent epithelial defect (PED) or recurrent erosions. LSCD may appear following exogenous factors: infections, chemical, or thermal burns, long-term use of topical medication, contact lenses, irradiation, tumors, surgery, and medical conditions, such as Steven-Johnson syndrome, aniridia or ocular pemphigoid $(5,6)$. LSCD can be unilateral or bilateral, partial or total, according to the extent of deficiency of stem cells. Partial LSCD means that there are regions where the population of stem cells is normal; therefore conjunctivalization occurs only in those regions where cells are insufficient. Total LSCD results in conjunctivalization of the entire cornea (7).

The management of corneal LSCD has changed over the years. The surgical treatment is dependent on the unilaterality/bilaterality of the condition. In 1989, Kenyon and Tseng (8) performed conjunctivolimbal autograft transplantation (CLAU). The tissue was harvested from the healthy or less damaged eye to treat a contralateral severe LSCD. For patients with bilateral LSCD Tsai and Tseng (9) performed keratolimbal allografting (KLAL). KLAL can successfully restore ocular surface stability in about $50 \%$ of bilateral ocular surface disease. Clinical outcome in KLAL is worse than in CLAU and the incidence of complications is higher (graft failure, repeated transplantation, glaucoma). Allografts are obtained either from a living related donor or a cadaver. Immunosuppression is needed in order to prevent the rejection of the graft. Immunosuppression is adapted individually, according to the severity of graft dysfunction, $A B O$ blood type of the patient, reactive antigen and repeated failure of the graft (10).

A more recent development for the management of LSCD is the transplantation of cultured stem cells. The cultured limbal stem cell transplant (CLET) starts with a millimeter-size limbal biopsy from an unaffected site if there is one $(9,11)$. The limbal biopsy contains populations of LESCs, which are cultivated to provide enough cultured cells for transplantation. One of the advantages of CLET is the use of a smaller limbal tissue. The complications are lower than in CLAU, and further biopsies are possible if needed.

The problem of bilateral total loss of LESCs is more difficult to solve. Autologous source of limbal stem cells is not available in this case. Stem cells can be provided then, by donors (allogenic LESCs), or can be found in other parts of the body (different types of stem cells). The success of the cultivated autografts encouraged the clinical use of allografts obtained from cadavers or living donors (allogenic CLET), in the case of total bilateral loss of LESCs. The problem of immunosuppression remains similar to KLAL but it was speculated that there might be a reduced risk of allograft rejection when using ex vivo cultivated cells, which could be explained by the absence of antigen-presenting Langerhan's cells (7).

To overcome the problems of allograft rejection, oral mucosal epithelium was also cultivated and transplanted with favorable results (cultivated oral mucosa epithelial transplant-COMET). Studies have shown that oral mucosa epithelial cells require a reduced time for cultivation and they remain non-keratinized for an extended period of time (12). CAOMECS (cultivated autologous oral mucosal epithelial cell sheet) as an alternative to restore the corneal surface is a newer version of COMET and it uses a culture system which is sensitive to temperature. The cultivated cell sheet can be transplanted without additional support or substrate $(13,14)$.

\section{OBJECTIVE}

In this review we examined the two procedures usually applied for the treatment of total bilateral stem cells deficiency, COMET/CAOMECS, and allogenic CLET. We compared the outcomes of the two types of surgeries, the clinical and anatomical rates of success, and the complications. To our knowledge, there is only 1 clinical study comparing the 2 procedures, and it was also included in this study (15).

\section{Literature Search}

This review included studies reporting clinical results following allogenic CLET and COMET/CAOMECS in the past 15 years, regardless of the age of patients or etiology of LSCD. We have explored all the published data in the recognized databases like PubMed, Science Direct, and Scopus by using the terms: "stem cell transplantation; cornea; (allogenic or allogeneic)" and "transplant; cornea; oral mucosa." In the searched period, from 2004 to 2019, we found 30 studies matching criteria, 18 clinical studies for allogenic CLET, with publication date ranging from 2005 to 2019, 11 clinical studies for COMET or CAOMECS, with publication date from 2004 to 2019, and 1 study comparing the two of them, from 2019. Studies comparing clinical results of CLET or COMET to other surgical techniques were also included, but only data referring to allogenic CLET or COMET were taken into consideration.

\section{LIMBAL STEM CELLS CULTURING TECHNIQUES}

Literature has described two systems for the ex vivo cultivation of LESC: the explant culture system and the suspension culture system. Previous research showed that the two culture systems provide similar results regarding stem cells content (16). The explant system used amniotic membrane, for providing growing substrate and for transporting the cultured cells (4). This process can take from 14 to 28 days. Parihar (17) used the explant technique, placing small pieces of tissue, cut from 2 by $2 \mathrm{~mm}$ limbal biopsies, onto denuded amniotic membrane. A more detailed view regarding the size of limbal biopsies is provided in Table 1. The suspension culture system used enzymes in order to separate epithelial cells, obtaining a suspension of cells. 
TABLE 1 | The size of limbal and oral mucosa biopsies in allogenic CLET and COMET/CAOMECS studies.

\begin{tabular}{|c|c|c|}
\hline Surgical procedure & Author, year & Size of grafted tissue \\
\hline \multirow[t]{19}{*}{ COMET } & Daya et al. (18) & $1-2 \mathrm{~mm}$ \\
\hline & Shimazaki et al. (19) & Not mentioned \\
\hline & Kawashima et al. (20) & $1 \times 3 \mathrm{~mm}$ \\
\hline & Shortt et al. (21) & $2-3 \mathrm{~mm}$ \\
\hline & Pauklin et al. (22) & $1 \times 2 \mathrm{~mm}$ \\
\hline & Basu et al. (23) & $2 \times 2 \mathrm{~mm}$ \\
\hline & Prabhasawat et al. (24) & $2 \times 2 / 3 \times 1 \mathrm{~mm}$ \\
\hline & Qi et al. (25) & Not mentioned \\
\hline & Shortt et al. (26) & Not mentioned \\
\hline & Zakaria et al. (27) & Not mentioned \\
\hline & Ramírez et al. (28) & $2 \times 2 \mathrm{~mm}$ \\
\hline & Ganger et al. (29) & $2 \times 2 \mathrm{~mm}$ \\
\hline & Parihar et al. (17) & $2 \times 2 \mathrm{~mm}$ \\
\hline & Chen et al. (30) & Not mentioned \\
\hline & Cheng et al. (31) & Not mentioned \\
\hline & Behaegel et al. (32) & $1 \times 2 \mathrm{~mm}$ \\
\hline & Borderie et al. (33) & $1 \mathrm{~mm}$ \\
\hline & Campbell et al. (34) & Not mentioned \\
\hline & Wang et al. (15) & Not mentioned \\
\hline \multirow[t]{12}{*}{ COMET/CAOMECS } & Nishida et al. (35) & $3 \times 3 \mathrm{~mm}$ \\
\hline & Chen et al. (36) & $6 \times 6 \mathrm{~mm}$ \\
\hline & Nakamura et al. (37) & Not mentioned \\
\hline & Satake et al. (38) & $8 \mathrm{~mm}$ punch \\
\hline & Priya et al. (39) & $4 \times 2 \mathrm{~mm}$ \\
\hline & Burillon et al. (13) & $3 \times 3 \mathrm{~mm}$ \\
\hline & Sotozono et al. (40) & $6 \mathrm{~mm}$ \\
\hline & Kolli et al. (41) & $3 \mathrm{~mm}$ \\
\hline & Kocaba (14) & $3 \times 3 \mathrm{~mm}$ \\
\hline & Dobrowolski et al. (42) & $3-5 \mathrm{~mm}^{2}$ \\
\hline & Kim et al. (43) & $0.8 \times 1.5-1 \times 2 \mathrm{~cm}^{2}$ \\
\hline & Wang et al. (15) & $4 \times 4 \mathrm{~mm}$ \\
\hline
\end{tabular}

1-2 $\mathrm{mm}$ long biopsies were trypsinized and introduced into a suspension culture system, establishing multiple cultures. Half of the corneo-scleral rim was used in order to achieve cells suitable for culture. The cells from the suspension were arranged on a culture dish, culture medium was added and then the cells were incubated between 14 and 21 days. After this period of time, when cells were prepared to be transplanted, carriers, such as contact lens, amniotic membrane, paraffin gauze, nylon or collagen shields, were used for transportation of stem cells to the ocular surface (7). Shimazaki et al. (19) used both techniques, explant and suspension.

The culture medium consisted of: complete growth medium of Dulbecco's modified Eagle's medium, F12, irradiated fetal bovine serum, hydrocortisone, cholera toxin, recombinant human insulin, epidermal growth factor and antibiotics $(18,21-$ 23 ). Some studies used autologous serum from the donor to make a xeno free transplant $(22,23)$. Epithelial cells were cultivated on a cryopreserved and partially decellularized amniotic membrane.
One study mounted the cells on a non-adherent nylon dressing (18). Cultivation success was defined when a monolayer of cells became confluent (17).

The tissue from a cadaveric donor was obtained within $24 \mathrm{~h}$ (22) to 7 days post-mortem (28). One study obtained cadaveric tissue within 2-3 weeks before the planned allogenic CLET procedure (24). Basu et al. (23) used only tissue from living donors, Daya et al. (18), Shimazaki et al. (19), and Pauklin et al. (22) used both sources (living donor and cadaveric). Corneoscleral rings discarded after corneal graft harvesting procedures were used in order to achieve cells suitable for culture.

Most culture protocols used 3T3 feeder cells in order for the graft to receive proper nutrients, but they also have a role in detoxifying the culture media and providing extracellular matrix proteins (44). Inactivated 3T3 fibroblast cells were previously described to enhance proliferation $(7,45)$. These feeder cells undergo inactivation using mitomycin $\mathrm{C}$ or irradiation. Because of their animal exposure during the culture process, there may be a higher risk of rejection and possible viral infection. Some protocols included human-derived feeder cells layers instead of the 3T3 feeder cells (46), because they were associated with a higher safety profile.

Some authors conducted morphological and immunohistochemical studies in order to determine the cellular morphology after cultivation $(45,46)$.

\section{ORAL MUCOSAL EPITHELIAL CULTIVATION}

After the oral cavity was sterilized, a specimen of oral mucosa was excised from the interior buccal mucosal epithelium (35). The tissue harvested varied in size-from 3 by $3 \mathrm{~mm}$ (35) to 10 by $20 \mathrm{~mm}$ (17). The size of oral mucosa biopsies is detailed in Table 1. Cell isolation and cultivation methods were similar to those described above. Cell suspension techniques were applied in all studies, with one exception (17).

\section{SURGICAL TRANSPLANTATION}

\section{Surgical Technique}

Preoperative preparations in most allogenic CLET studies included tissue screening for human immunodeficiency virus 1 and 2, syphilis, hepatitis B and C (21-23, 28, 29). Cheng et al. documented a similar screening protocol but did not include testing for syphilis (31). Authors from two studies also tested the tissue for human T cell leukemia-lymphoma virus $(21,28)$. One study refers to using a previous protocol where preoperative screening was performed (26). The other CLET studies which we included in this review did not mention screening protocols (20).

The transplantation procedure included a $360^{\circ}$ conjunctival peritomy and removal of the modified corneal epithelium. The cultivated limbal stem cells were transferred onto the ocular surface on amniotic membrane, which was secured with sutures in all studies with two exceptions, Zakaria et al. (27), who secured the graft with fibrin glue, and Ganger et al. (29), who 
used both, either sutures or fibrin glue. In some studies a bandage contact lens was inserted after the transplant $(21,24,26-$ 29,31 ) while in other studies a second amniotic membrane was used as a patch $(18,22,27,34)$. One study mentioned using either bandage contact lens or amniotic membrane (20) whereas one study does not mention the use of either (17) In cases of symblepharon, fibrovascular tissue surrounding rectus muscles was dissected in order to achieve normal ocular movement (31).

In COMET and CAOMECS, the transplantation technique was similar to allogenic CLET. The cultivated oral mucosal epithelial sheets were transferred to the corneal surface after removing the modified epithelium. In most cases, cultured sheets were not secured with sutures $(13,14,35,37,43)$, however a therapeutic contact lens was used over the transplant. Nonetheless, a few authors reported suturing cultivated sheets to the conjunctiva $(36,38,42)$. With patients also suffering from cataract, phacoemulsification and posterior chamber implant were performed simultaneously with COMET (40). Kolli et al. (41) does not mention the exact transplantation technique. Priya et al. (39) additionally used mitomycin C for subconjunctival spaces.

\section{Post-operative Considerations}

Post-operative management mainly included topical antibiotics, immunosuppression and lubricating eye drops and systemic immunosuppression. Post-operative therapy for all allogenic CLET studies included topical antibiotics (ciprofloxacin $0.3 \%$, tobramycin $0.3 \%$, ofloxacin $3 \%$, levofloxacin or chloramphenicol $0.5 \%)$ with two exceptions $(19,26)$. Local immunosuppression was obtained by topical prednisolone acetate $1 \%$, fluorometholone $0.02 \%$, dexamethasone $1 \% / 0.1 \%$, or methylprednisolone 1\%. Cyclosporine A drops were included as topical regimen in two studies $(19,31)$. On the 1 days after surgery, patients were administered oral immunosuppression agents. Systemic immunosuppression was obtained by Cyclosporine A and steroids. One study described using mycophelonate mofetil and azathioprine also (28). In one case, a patient received mycophenolate mofetil, due to the fact that cyclosporine A was not tolerated (22). Autologous $20 \%$ serum eye drops were also included in the 1 week post-operative by some authors $(18,22,24,27,31)$. One study documented inducing ptosis in all patients during the 1 week following CLET (22). In all studies, immunosuppression was slowly tapered within months after the transplant.

A study which analyzed the outcomes of allogenic CLET and DNA analysis of donor, suggested that immunosupression more than 9 months following CLET might be not be needed (18).

Similarly, following COMET/CAOMECS, patients received antibiotics and anti-inflammatory agents [topical or general corticosteroids, or Cyclosporine (37)], in order to keep inflammation to the minimum, and artificial tears $(13,14,35-$ $38,40-43)$. In some studies, autologous serum drops were used also $(41,42,47)$.

\section{CLINICAL OUTCOMES}

Clinical outcomes following allogenic CLET and COMET/CAOMECS for bilateral limbal stem cell deficiency of different etiologies are summarized in Table 2, in chronological order. There is no common view regarding the assessment of the clinical success. The outcomes of the surgery were mostly evaluated based on clinical and functional findings. Impression cytology or confocal microscopy, as outcome measures, were described by Shortt el al. $(21,26)$. Impression cytology alone was used by Daya et al. in order to perform DNA analysis (18) and by Prabhasawat et al. (24). Shimazaki et al. (19) used impression cytology in order to confirm conjunctivalization preoperatively and to asses corneal phenotype following surgery. In vivo confocal microscopy was used by Ramirez et al. to assess epithelial phenotype of the central cornea 1 year following CLET (28).

\section{Allogenic CLET Studies}

Daya et al. (18) reported an improvement of LSCD in 7 patients, $70 \%$ of the cases. All were severe LSCD, with four quadrants involvement. Mean follow up was 28 months. Parameters followed were vascularization, conjunctivalization, inflammation, epithelial defect, photophobia and pain. The seven patients considered a success also had performed impression cytology with DNA analysis. This revealed no donor DNA present on ocular surface in 5 out of the 7 cases tested between 1 and 7 months post-op. The other two cases had positive donor DNA present at 2 and 7 months, respectively, but not after 9 months. Four cases (40\%) also had an improvement in visual acuity. Three of these cases underwent perforating keratoplasty (PK) and supplemental KLAL at different intervals after the main surgery. Three other patients received KLAL together with forms of keratoplasty (PK, DALK, lamellar) and amniotic membrane transplantation. Second procedures were performed to improve vision of for tectonic reasons at 5-40 months after the first surgery. KLAL was supposed to act as a barrier for conjunctivalization. Best visual acuity was $20 / 25$ but the other three cases reached 10/100, 20/100, and 20/80.

Shimazaki et al. (19) reported $62.5 \%$ success rate for stem cells provided by living relatives donors and $41.7 \%$ for unmatched cadaveric donors. Their study included 27 eyes from 27 patients and the mean follow-up time was 127 weeks. Success in ocular surface reconstruction was defined as having stable epithelium with corneal phenotype on the central cornea with and without peripheral conjunctival invasion. Corneal phenotype was evaluated with impression cytology or slit-lamp examination with fluorescein staining. There were complications reported but the report did not differentiate between autologous and allogenic technique.

Kawashima et al. (20) evaluated cell phenotype of the central cornea post-operative. Slit lamp examination and fluorescein staining showed corneal phenotype after allogenic CLET surgery. Histopathological examination revealed corneal phenotype in 4 of 6 patients. Epithelial cells were positive for $\mathrm{K} 3$ and negative for $\mathrm{K} 13$, which indicated the presence of normal corneal epithelium. The follow-up period ranged from 5 to 
TABLE 2 | Results following CLET and COMET.

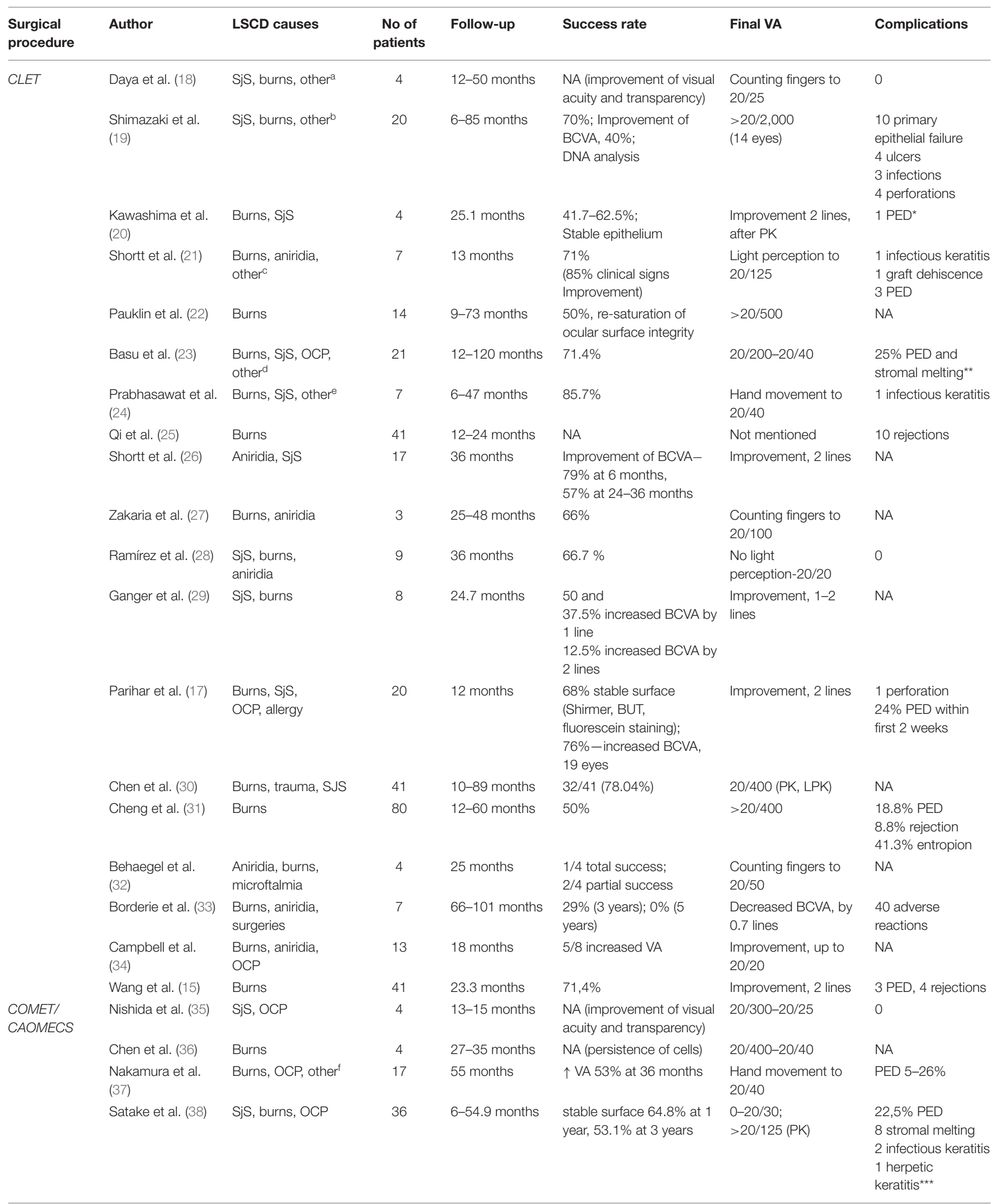


TABLE 2 | Continued

\begin{tabular}{|c|c|c|c|c|c|c|c|}
\hline $\begin{array}{l}\text { Surgical } \\
\text { procedure }\end{array}$ & Author & LSCD causes & $\begin{array}{c}\text { No of } \\
\text { patients }\end{array}$ & Follow-up & Success rate & Final VA & Complications \\
\hline & Priya et al. (39) & Burns, SJS & 7 & 1-34 months & $\begin{array}{l}3 / 7(42 \%) \text { anatomical; } \\
\text { from which } 2 \text { with visual } \\
\text { improvement }(28 \%)\end{array}$ & $\begin{array}{l}\text { Light perception to } \\
20 / 200\end{array}$ & 2 rejections \\
\hline & Burillon et al. $(13)^{\#}$ & $\begin{array}{l}\text { Burns, aniridia, } \\
\text { other }^{9}\end{array}$ & 26 & 12 months & $75 \%$ (16 patients) & $\begin{array}{l}\text { Hand movement to } \\
\text { 020/50 }\end{array}$ & $\begin{array}{l}1 \text { perforation } \\
1 \text { rejection }\end{array}$ \\
\hline & $\begin{array}{l}\text { Sotozono et al. } \\
(40)\end{array}$ & $\begin{array}{l}\text { Burns, SjS, OCP, } \\
\text { other }^{\text {h }}\end{array}$ & 40 & $\begin{array}{l}6.2-85.6 \\
\text { months }\end{array}$ & $\begin{array}{l}\text { Improved BCVA: } \\
50 \% \text {-SJS, } \\
\text { 42,9\%-OCP, } \\
20 \% \text { - burns }\end{array}$ & $\begin{array}{l}\text { Improvement, at least } \\
1 / 2 \text { line }\end{array}$ & $\begin{array}{l}40 \% \text { PED, } \\
5 \% \text { stromal melting, } \\
5 \% \text { slight-mod } \\
\text { infection }\end{array}$ \\
\hline & Kolli et al. (41) & Burns & 2 & 21-41 months & NA (stable epithelium) & 20/200-20/63 & NA \\
\hline & Kocaba $(14)^{\#}$ & $\begin{array}{l}\text { Burns, aniridia, } \\
\text { other }\end{array}$ & 23 & 28 months & Improved BCVA 74\% & Improvement, 2.3 lines & $\begin{array}{l}1 \text { perforation } 1 \\
\text { rejection }\end{array}$ \\
\hline & $\begin{array}{l}\text { Dobrowolski et al. } \\
\text { (42) }\end{array}$ & Aniridia & 13 & 12-18 months & $\begin{array}{l}\text { 76.4\% stable surface; } \\
\text { Improved BCVA 88.2\% }\end{array}$ & $\begin{array}{l}\text { Hand } \\
\text { movement-20/200 }\end{array}$ & 3 graft failures \\
\hline & Kim et al. (43) & Burns, SjS & 8 & 2-15 months & $75 \%$ & Improvement $>2$ lines & $50 \%$ PED \\
\hline & Wang et al. (15) & Burns & 32 & 16.1 months & $52.9 \%$ & $\begin{array}{l}\text { Decreased } \\
\text {-improvement } 2 \text { lines }\end{array}$ & $\begin{array}{l}9 \text { PED, } 5 \text { stromal } \\
\text { melting }\end{array}$ \\
\hline
\end{tabular}

\# CAOMECS.

${ }^{\star}$ Complications post-PK: 2 endothelial rejections (months 18-24).

${ }^{\star *}$ Complications post-PK: $69.2 \%$ graft failure, 33.3\% endothelial rejection, $11.1 \%$ traumatic dehiscence, $22.2 \%$ recurrence of LSCD.

${ }^{\star \star \star}$ Complications post-PK: 1 perforation, 1 endothelial rejection.

SjS, Sjogren Syndrome; OCP, ocular cicatricial pemphigoid; PED, persistent epithelial defect; NA, not available; BCVA, best corrected visual acuity.

${ }^{a}$ Rosacea blepharoconjunctivitis, ectodermal dysplasia.

${ }^{b} \mathrm{OCP}$, keratoconjunctivitis, unknown cause.

${ }^{c}$ Ectodermal dysplasia, Reiger's anomaly.

${ }^{d}$ Allergy, contact lens hypoxia.

${ }^{\text {e} A l l e r g y, ~ d r y ~ e y e ~ s y n d r o m e, ~ m u l t i p l e ~ e y e ~ s u r g e r i e s . ~}$

${ }^{f}$ Squamous cell carcinoma, graft vs. host disease.

${ }^{g}$ Contact lens hypoxia, Lyell syndrome, rosacea keratitis, neuroparalytic keratitis, Groenow dystrophy, trachoma, hepatitis C, cystinosis.

${ }^{h}$ Graft vs. host disease, Salzmanns degeneration, radiation keratopathy, drug induced LSCD, idiopathic LSCD.

${ }^{i}$ Contact lens hypoxia, Lyell syndrome, rosacea keratitis, neuroparalytic keratitis, Groenow dystrophy, trachoma, hepatitis C, cystinosis.

41 months. A decrease of the neovascularization was also recorded. Visual acuity was not significantly improved due to persistent stromal opacification, however, it was improved when additional procedures were performed. One case of persistent epithelial defect was present, but it responded well to topical treatment.

Ramirez et al. (28) studied post-operative results by dividing patients into three categories: burns, inflammations related to autoimmunity and non-inflammatory diseases. Success rate was $66.7 \%$ at the 1 year follow-up for patients who underwent allogenic CLET. In vivo confocal microscopy performed at the 12 months follow-up was used to determine the presence of corneal phenotype after CLET. Following allogenic CLET, five cases which initially were allocated to having conjunctival-like or mixed epithelial cell phenotype, had improved to corneal phenotype. One patient presented mixed phenotype. No adverse reactions or rejection was recorded.

The study carried out by Ganger et al. (29) mainly focused on results of allogenic transplant in children compared to adults. The mean follow-up time was 24.7 months. Out of the eight patients, five were children (patients under the age of 15) and three were adults. A successful surgery was defined by anatomical and visual acuity improvement. Anatomical success was observed in $50 \%$ of the cases and visual acuity was improved by one line in $37.5 \%$ of cases and by 2 lines in $12.5 \%$ of cases. Nonetheless, comparison of the results between the two groups was not made due to the small amount of patients.

Shortt et al. (21) studied eight cases of allogenic transplant and reported an improvement of clinical signs in $85 \%$ of the them. A successful surgery was defined by improvement of the visual acuity, absence of signs of LSCD, corneal phenotype on impression cytology and presence of corneal cells at confocal microscopy. At the 6 months follow-up patients had increased corneal transparency, reduced or absent corneal vascularization and the corneal surface was smooth. Impression cytology and confocal was used to evaluate the presence of corneal morphology. In impression cytology, samples were immunostained with monoclonal antibodies in order to evaluate the phenotype of the cells from the corneal surface. Results were compared preoperative and post-operative. Corneal phenotype was present in $5 / 7$ of the samples. There were only few complications: one graft failure was reported and a secondary transplant was performed. The overall success rate of CLET was $71 \%$.

Pauklin et al. (22) considered that a completely restored ocular surface was the major success of the surgery and it was achieved in $50 \%$ of the cases. The mean follow-up time was 
28.5 months. Improvement of preoperative and post-operative visual acuity was considered less important, however it was documented in $64.3 \%$ of the cases. In eyes which previously suffered a chemical or thermal injury, the visual acuity improved the most. A successful surgery meant a smooth and clear corneal surface, without epithelial defects and without recurrence of conjunctivalization. Failure of surgery was represented by loss of transparency of the corneal surface, superficial vascularization in more than one quadrant and persistent epithelial defects and was documented in 4 eyes (partial success in 4 eyes).

A stable corneal surface was also considered a success by Basu et al. (23) and it was reported in $71.4 \%$ of the cases. LSCD was defined as $360^{\circ}$ superficial corneal vascularization, diffuse fluorescein staining of the corneal surface with or without persistent epithelial defects, conjunctivalization of the corneal surface and absence of limbal palisades of Vogt. The recurrence of epithelial defects or superficial corneal vascularization was defined as failure of surgery. Measurement of visual acuity and evaluation of possible complications were also studied. Although VA improved in all 28 eyes, failure as defined was documented in $8 / 28$ eyes (28\%; $75 \%$ of the failures were between 1 and 9 months). Some complications were related to the instability of the corneal surface, whereas others were related to the immunosuppressive treatment. PK was performed in 13 eyes 14 months (average duration between allogenic CLET and PK was 12-22 months) after CLET if the VA was worse than 20/60 and attributed to corneal stromal scarring. Authors did not mention the followup time post-PK but the mean follow-up time after CLET was 4.8 years. Histology performed on the excised cornea showed no sign of amniotic membrane or goblet cells and epithelial cells expressed cornea specific marker K12.

While a stable corneal surface was the main outcome measured by most authors, Prabhasawat et al. (24) also included histological outcomes to determine success after allogenic CLET. A good histological outcome was characterized by the absence of goblet cells in the central cornea. Interestingly, the success rate was higher in the allogenic group. The reported success rate was $85.7 \%$ after allogenic CLET vs. $66.7 \%$ after autologous CLET. However, lid abnormalities, more common in the autograft group, could explain the results. There was one graft failure due to infectious keratitis.

Zakaria et al. (27) assessed both anatomical and functional success after CLET. Improvement of pain, photophobia and visual acuity was a functional success. They reported 2 out of 3 successful cases following allogenic CLET. There was one failed case, which presented a lack of epithelialization of the corneal surface and conjunctivalization.

Parihar et al. (17) described improvement of visual acuity after CLET and lack of conjunctivalization in 12 out of 20 patients $(60 \%)$. At the 1 year follow-up, in 14 out of 24 eyes (58.33\%) there was no conjunctivalization noted. Ocular surface stability was evaluated by Schirmer test, BUT and fluorescein staining. However, persistent epithelial defects were recorded in 6 cases within 2 weeks after surgery. One case of perforation following allogenic CLET and some cases of adverse reactions due to immunosuppressive therapy were reported.
Cheng et al. (31) evaluated primary and secondary outcomes of CLET in 80 cases of symblepharon, after a follow-up of 1260 months. Complete success was represented by the absence of scars or ocular motility restriction and the presence of a deep conjunctival fornix. Secondary outcomes were evaluated based on: visual acuity at follow-up compared to visual acuity preoperative, presence of complications, risk factors for the recurrence of symblepharon and surgery for recurrent symblepharon. The success of the surgery was associated with the severity and the inflammation of the symblepharon. The complete success rate was 50\% after first surgery (40 eyes), partial success was $31.3 \%$, and failure was $18.8 \%$ (15 eyes). Corneal condition (diminished conjunctivalization) improved in 43 eyes (53.8\%). The cause of the symblepharon had an influence on the outcome of the surgery. In patients with symblepharon following thermal injuries, success was achieved in 31 out of 44 cases. The success rate was higher in cases of symblepharon following chemical injuries, 34 out of 36 cases $-94.4 \%$. Recurrent symblepharon was documented in $50 \%$ of cases during the first 3 months post-operatively. Eyes with thermal injuries recorded a more rapid recurrence. Complications, such as immune rejection or recurrent epithelial defects were recorded. Nonetheless, a grading system would be required for a better evaluation of post-operative outcomes. In 2014, Shortt et al. (26) tried to design a grading system for a better and objective assessment of results. Clinical signs related to LSCD that were assessed by previous studies were included in the grading system. The most relevant four clinical signs were included: corneal epithelial haze, epithelial defect, irregularity of the epithelium and superficial vascularization. Each of these parameters was attributed with grades from 0 (normal) to 3 (severe). Photographs of the cornea were also included, which were taken according to specific parameters (high magnification $\times 16$ of the central cornea, under blue cobalt illumination after one drop of fluorescein $2 \%$ was instilled). Based on the grades given to the clinical signs and photographs, the severity (which ranged from 0 to 12 ) was analyzed. This grading system is titled The Clinical Outcome Assessment in Surgical Trials of Limbal stem cell deficiency-COASTL. With the use of COASTL grading system, each case was graded a global score, based on clinical parameters, which allowed a better and objective evaluation. However, to our knowledge this grading system was not used in other studies. This study included cases of aniridia and Stevens Johnson syndrome. Improvement of visual acuity was documented in $79 \%$ of the eyes at the 6 months follow-up, $71 \%$ of eyes at the 12 months follow-up and $64 \%$ of eyes at the 18 months follow-up. In patients with aniridia, improvement of the global score was noted 12 months after CLET. LSCD associated with Stevens Johnson syndrome recorded an improvement 6 months after surgery, and then between 6 and 18 months post-operatively signs of LSCD were observed. After 18 months, a stabilization and improvement was again documented.

Borderie et al. (33) aimed to compare outcomes following cultured LESCs transplantation to limbal tissue transplantation. They included 30 eyes with LSCD stage III, which is characterized by vascularization of the entire cornea, irregular epithelium, 
staining of the entire limbus and central cornea. Seven patients underwent allogenic CLET and 8 underwent allogenic limbal transplantation. The survival of the graft at the 3-years mark was 29\% for allogenic CLET, 50\% for allogenic limbal transplantation and $0 \%$, respectively, $33 \%$ at 5 years following surgery. A reduction in visual acuity was recorded ( 0.7 lines for allogenic CLET and 1.9 lines for allogenic limbal tissue transplantation).

Behaegel et al. (32) followed four patients which underwent allogenic CLET for a mean period of 2.1 years to establish the short and long term results of surgery. Long term results showed that all cases could either be categorized as partial success or failure.

Campbell et al. (34) conducted a randomized clinical study and distributed the patients into two groups: one which received allogenic corneal epithelial stem cells on amniotic membrane and one which received only amniotic membrane. Both groups received immunosuppression. Sixteen patients were included but only 13 remained at the end of the follow-up period. The mean follow-up was 32 months. Improvement in visual acuity was recorded in both groups; however, in these cases cataract surgery was also performed. Ocular surface was more stable and received a lower score in the group of allogenic CLET. The main purpose of the study was to establish if there is a long-standing and superior outcome post-transplant and concluded that allogenic CLET is feasible and safe procedure in severe bilateral LSCD.

The characteristics of immune rejection following allogenic CLET was studied by Qi et al. (25). They included 41 patients in their study and the recorded immune rejection rate was $23.8 \%$ (10 eyes, 9 patients). Mean duration between surgery and immune rejection was 3.1 months. In one case, they could identify a causative factor: the patient ceased the topical 1\% Cyclosporine A treatment. Apart from the clinical features of immune rejection (epithelial edema, epithelial rejection lines, persistent peripheral epithelial defects in six eyes, aggravation of vascularization in eight eyes and stromal opacity in nine eyes), impression cytology was performed in six cases and it revealed $\mathrm{CD} 4^{+}$and $\mathrm{CD} 8^{+}$ cells. In vivo confocal microscopy demonstrated the presence of network-like Langerhans cells in central and peripheral cornea. All eyes were however restored to a stable ocular surface with anti rejection treatment.

The corneal surface following allogenic CLET was analyzed by Chen et al. (30), who included 41 eyes of 41 patients. The mean follow-up time was 22.13 months. Clinical success was recorded in 32/41 eyes (78\%). Authors analyzed by immunohistochemical staining 41 pannus specimens and found positive staining for K19 in all specimens. Sporadic staining for K3 and P63 was also recorded. Histology also revealed a higher number of cell layers compared to normal corneal epithelium. They also performed impression cytology and subsequent PCR and STR analysis for 14 pannus specimens and 5 corneal buttons in order to establish if DNA donor was present after 3 months. All specimens were negative for DNA donor. Therefore, speculation that the multilayered epithelium could be residual cultured cells was invalidated. Authors speculated that transplanted cultured cells could be affected by a chronic immune rejection and accumulation of inflammatory cytokines. They also speculated that a residual number of residual stem cells of the host are stimulated to proliferate, therefore ensuring stability to the ocular surface.

Wang et al. (15) compared results of allogenic CLET to COMET. Seventy-six eyes of 73 patients were included: 41 patients (42 eyes) in the allogenic CLET group and 32 patients (34 eyes) in the COMET group. Mean follow-up was 23.3 months for allogenic CLET and 16.1 months for COMET. A higher success rate was recorded in the allogenic CLET group compared to COMET (71.4\%, respectively, 52.9\%, $p=$ 0.043). Authors found higher failure rate that was associated with eyelid abnormalities and following COMET (3.5 times higher than allogenic CLET). The complications following allogenic CLET were PED (three cases), recurrent symblepharon (four cases), recurrent corneal conjunctivalization (four cases), and immune rejection (one case). In the COMET group, nine had PED, four had recurrent symblepharon, and three cases had recurrent corneal conjunctivalization. In allogenic CLET, $47.6 \%$ showed improved vision by one line ore more (compared to 50\% in COMET), while two eyes (4.8\%) worsened (compared to COMET, three eyes worsened, 8.8\%) and the rest showed no improvement. There was no statistically significant difference in preoperative and post-operative vision between the two groups. Although allogenic CLET poses a rejection risk, anti rejection therapy was effective, and graft failure due to rejection was rare. The authors suggested that, because patients treated with COMET were more likely to develop persistent epithelial defects, leading to graft failure, allogenic CLET should be prioritized.

\section{COMET/CAOMECS Studies}

Nishida et al. (35) reported in 2004 complete re-epithelization at 1 week following COMET and the central corneal surface maintained its transparency during the 14 months follow-up period. On the other hand, the rate of success was not mentioned in the study and authors did not report any complications.

Nakamura et al. (37) evaluated clinical results (conjunctivalization, corneal opacification, neovascularization and symblepharon formation) following COMET according to their grading system. Improvement of more than one line of visual acuity was documented in 53\% of cases at the 36 months follow-up. Out of 19 eyes which received COMET, 7 eyes (37\%) were documented with at least one episode of persistent epithelial defect during the follow-up period and three eyes (16\%) presented ocular hypertension.

The main outcomes of Satake et al. (38) were a transparent corneal surface, without fibrovascular invasion and a functional fornix. Improvement of visual acuity was also recorded but additional surgery (keratoplasty) was required for better results than COMET alone. Complications like fibrovascular invasion were found in $20 \%$ of cases and persistent epithelial defects were present in $22.5 \%$ of patients, with a higher rate among limbal stem cell deficiency following chemical or thermal burns and Stevens-Johnson syndrome.

Following CAOMECS, a success rate of $75 \%$ was reported by Burillon et al. (13) during the 12 months follow-up period. Patients also had an improvement as far as photophobia, pain and 
visual acuity were concerned. Two patients out of the 25 included in the study were classified as graft failures. Sotonozo et al. (40) mainly focused on the improvement of visual acuity following COMET and concluded that this was influenced by factors like: preoperative visual acuity, etiology of LSCD, age of patient, two-step surgery, concomitant cataract surgery or transplant of amniotic membrane. Visual prognosis was better in cases of severe neovascularization. They described the rate of success regarding visual acuity according to the etiology. They included 40 patients in their study, 17 cases of Stevens-Johnson syndrome, nine patients with ocular cicatricial pemphigoid, 6 cases of chemical/thermal injury and eight patients with other causes of limbal stem cell deficiency. They recorded an improvement in $50 \%$ of cases suffering from Stevens-Johnson Syndrome, $42.9 \%$ for ocular cicatricial pemphigoid and 20\% for LSCD following burns. However, authors mentioned that $48.9 \%$ of the patients included in the study had previously unsuccessful corneal or amniotic membrane transplantation. In $40 \%$ of cases there was a persistent epithelial defect following COMET and 5\% of patients presented with corneal melting, however, no perforation was recorded.

Kolli et al. (41) followed two patients after COMET and in both cases they documented a stable epithelium, improvement in visual acuity and regarding ocular pain and discomfort.

Kocaba et al. (14) studied the long term results of CAOMECS in bilateral LSCD and documented an improvement regarding visual acuity in $74 \%$ of cases. Furthermore, the 23 patients included in their study were divided into two groups: one which underwent corneal transplantation and one which did not. Mean follow-up duration was 28 months. There was no significant difference between the two groups regarding further visual acuity improvement. The authors did not mention the interval between CAOMECS and penetrating keratoplasty. Two graft failures were reported in the group which underwent corneal transplantation.

Dobrowolski et al. (42) studied 13 cases of aniridia patients in which a stable corneal epithelium was observed during the observation period of $12-18$ months. There were 3 cases of stromal scarring and vascularization and conjunctival vascularization. These cases were classified as graft failure.

Kim et al. (43) in a prospective clinical trial which studied the survival of COMET reported a rate of success of $75 \%$ and improvement in visual acuity in $62,5 \%$ of the cases at the 6 months follow-up. Their study included eight subjects, age ranging from 17 to 60 years. In four cases, penetrating keratoplasty was performed for residual stromal opacity following COMECs, after obtaining a stable corneal surface for a minimum of 6 months. Persistent epithelial defects were present in $50 \%$ of the cases at follow-up and were treated with amniotic membrane transplantation or semi scleral lens. No other adverse reactions were recorded.

Priya et al. (39) found a success rate lower than mentioned previous studies. The study included 10 male patients with bilateral chemical injuries or Stevens Johnson syndrome. Patients aged 8-65 years old were followed for a mean duration of 18.6 months. Anatomical success was recorded in $3 / 7$ patients $(42 \%)$ and visual improvement was present in $2 / 7$ cases $(28 \%)$.

\section{DISCUSSIONS}

The time distribution of clinical studies for CLET and COMET shows a homogenous interest in both of the procedures during the past 15 years (Figure 1). However, the number of patients included was very low, with mostly small series ranging from 2 to 40 patients. One exception was the study of Cheng et al. (31), which followed 80 patients with symblepharon for more than 12 moths.

A comparison between CLET and COMET/CAOMECS, regarding the results, is not fully possible. The small number of patients is divided between various pathologies with very different outcome: Sjogren Syndrome, burns (chemical or physical), ocular cicatricial pemphigoid, persistent epithelial defects, or aniridia. Even in CLET the method of cultivation was not consistent, some authors using explants only, others suspension cultures and others using both of them (19). The success of the graft is further influenced by the quality of donor material. Both cadaver and living matched donors were used, with similar outcomes. The success of the graft depends on whether the number of cultivated cells is sufficient or not. During ex vivo culture, only some limbal epithelial progenitors migrate onto the amniotic membrane. Some of the cells undergo mesenchymal transition (48). There was no difference between the methods of cultivation reported. Both explants and suspension cultures showed similar results. Zakaria et al. (27) also showed the viability of a xeno-free cultivation technique.

To further complicate the evaluation, the graft success was quantified by different criteria, from DNA analysis (18), impression cytology $(7,18,19,24)$, confocal microscopy (28) to clinical evaluation (visual acuity improvement, lack of vascularization of the cornea or stable epithelium). Sometimes the visual acuity was not improved although the ocular surface showed signs of improvement. These cases usually underwent additional surgical procedures, for instance corneal transplants. COMET or allogenic CLET seem to improve the outcome of such additional procedures (37).

Corneal healing is a fascinating topic although poorly understood. There is no precise mechanism for the function of the grafted stem cells. It is possible that some residual stem cells in total LCSD still remain in dormant state and are stimulated by donor stem cells to multiply (18). Donor stem cells seem to vanish few months after transplantation. In 2005, Daya et al. (18) first noted that after about 9 months there were no more donor cells and no DNA from the donor to be found in the allogenic CLET area. That suggested that CLET has no clear need of autologous source of stem cells or a well-matched living relative, as the cells will be totally replaced months after the grafting. Non-matched cadaveric stem cells from discarded limbal rings could be an adequate source. This could explain the lack o difference between the clinical outcomes from allogenic CLET and COMET, both with more than $50 \%$ rate of clinical success. Different authors showed that cells covering the cornea retain a corneal phenotype. Basu et al. (23) found no goblet cells in the successful grafts and epithelial cells expressed specific K12 corneal marker.

Because of autologous origin and lack of immune rejection, mucosal stem cells are expected to perform better than 


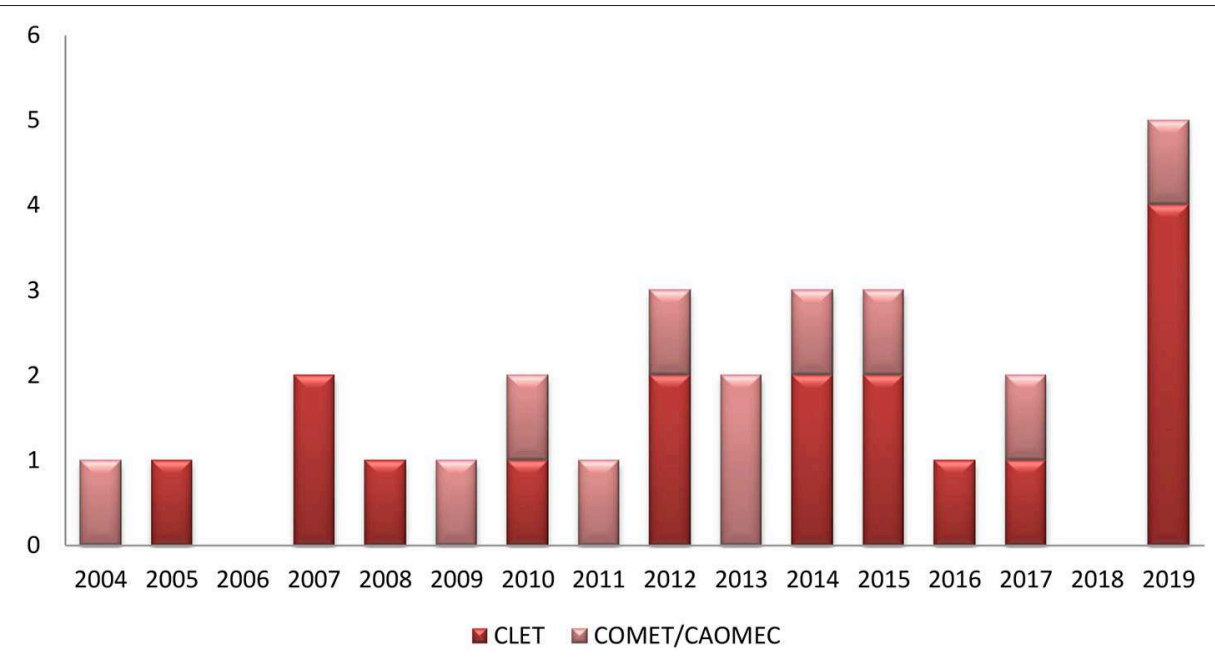

FIGURE 1 | Distribution of clinical studies from 2004 to 2019; comparison between CLET and COMET/CAOMECS.

allogenic stem cells. However, Wang et al. (15) showed that the morphology, function, and microenvironment of oral mucosal epithelium are very different from those of limbal epithelium, despite the expression of similar gene markers. They observed a higher incidence of persistent epithelial defects in COMEC than allogenic CLET.

As far as surgery success according to individual factors is concerned, it is difficult to draw conclusions from the studies mentioned in this review. Because of the heterogeneity of the data included in the studies, it is difficult to establish whether age, gender or cause of the limbal stem cell deficiency can influence the survival of the graft and success rate following surgery.

Shimazaki et al. (19) found a higher success rate in patients suffering from Stevens-Johnson syndrome, however they did not study individual factors. Satake et al. (38) also studied the rate of success according to the cause of LSCD. Patients with Stevens-Johnson syndrome had a more stable epithelium after CLET than patients with LSCD following burns or OCP. In one study which studied results of CLET in patients with aniridia, 3 out of the 13 female patients included had a lower rate of epithelium regularity (42). Ganger et al. (29) followed the longterm outcomes in children and adults following CLET, however comparison between the two groups was not done due to the small number of patients.

Following COMET, one study detailed results according to the disease. They concluded that patients with Stevens-Johnson syndrome had the greatest improvement in visual acuity. In patients with OCP and age over 60 years improvement in visual acuity was the best at 4 weeks post-operative but it later diminished (40).

One meta-analysis (49) also concluded that success rates are difficult to be compared due to the heterogeneity of the data, however, local factors, such as adnexal pathology, should be corrected for a better outcome.

Future perspectives for treating total bilateral limbal stem cell deficiency are currently under evaluation. Studies have shown promising results regarding using different cell sources. Hair follicle bulge-derived stem cells provided a good corneal surface after transplantation in murine models. Human immature dental pulp stem cells, embryonic stem cells and umbilical cord stem cells also showed good results in animal models (50, 51). One study (52) compared results between allogenic CLET and allogenic bone marrow-derived mesenchymal transplant (MSCT). They included 11 patients who underwent allogenic CLET and 17 who had MSCT. After a follow-up period of 6-12 months, they concluded that both surgical techniques had similar rates of success, $77.8 \%$ CLET, respectively, $85.7 \%$ MSCT.

Somatic cell reprogramming by using viral vectors was first evidenced by Chakrabarty et al. (53) resulting induced pluripotent stem cells (iPS). Even though iPSC might play a major role in regenerative medicine, there are a few concerns regarding their use. They carry an important risk of genomic instability which might influence their clinical applicability. Other issues related to their use are the potential tumorigenicity and the necessity of a longer cultivation period. The culture duration is dependent on the age of the donor, the phenotype of the reprogrammed somatic cell, and the cultivation conditions. The major advantage is the absence of immune rejection. The potential of iPS has started to be explored in human subjects. In Japan, Nishida described one of the first patients treated with iPS, with promising results (54).

\section{CONCLUSIONS}

The aim of our review was to summarize clinical outcomes following allogenic CLET and COMEC/CAOMECS. The visual acuity improvement in both methods was reported similar, with percentages usually higher than $50 \%$ of the treated cases. However, the comparison between studies was difficult to achieve due to the lack of a universal and objective grading tool for assessing post-operative results. Moreover, some studies 
which reported results following both auto and allogenic CLET, described mixed results for both procedures, therefore analyzing the results separately was impossible. As a further matter, only one study compared preoperative and post-operative aspects of limbal stem cell deficiency using in vivo confocal microscopy and impression cytology.

The differences in complication rate were difficult to assess due the fact that studies evaluated differently their outcomes. It was difficult to describe the impact of individual factors, such as age, gender, or cause of LSCD on the surgical outcomes due to the lack of sufficient data. COMET/CAOMECS may present certain advantages compared to CLET. These advantages are represented by overcoming the problems associated with allograft rejection, the achievement of cell culture in a shorter period of time and the absence of

\section{REFERENCES}

1. Nubile M, Curcio C, Dua HS, Calienno R, Lanzini M, Iezzi M, et al. Pathological changes of the anatomical structure and markers of the limbal stem cell niche due to inflammation. Mol Vis. (2013) 19:516-25.

2. Gonzalez G, Sasamoto Y, Ksander BR, Frank MH, Frank NY. Limbal Stem Cells: Identity, Developmental Origin, and Therapeutic Potential. Vol. 7. Wiley Interdisciplinary Reviews: Developmental Biology. John Wiley and Sons Inc (2018). doi: 10.1002/wdev.303

3. Atallah MR, Palioura S, Perez VL, Amescua G. Limbal stem cell transplantation: current perspectives. Clin Ophthalmol. (2016) 10:593-602. doi: 10.2147/OPTH.S83676

4. Samoilǎ O, Soritǎu O, Totu L, Suşman S, Mihu CM. Cultivation and characterization of limbal epithelial stem cells in rabbits. Rom J Morphol Embryol. (2014) 55:63-69. doi: 10.1097/ICO.0000000000000002

5. Liang L, Sheha H, Li J, Tseng SCG. Limbal stem cell transplantation: new progresses and challenges. Eye. (2009) 23:1946-53. doi: 10.1038/eye.2008.379

6. Yin J, Jurkunas U. Limbal stem cell transplantation and complications. Semin Ophthalmol. (2018) 33:134-41. doi: 10.1080/08820538.2017.1353834

7. Shortt AJ, Secker GA, Notara MD, Limb GA, Khaw PT, Tuft SJ, et al. Transplantation of ex vivo cultured limbal epithelial stem cells: a review of techniques and clinical results. Surv Ophthalmol. (2007) 52:483-502. doi: 10.1016/j.survophthal.2007.06.013

8. Kenyon KR, Tseng SC. Limbal autograft transplantation for ocular surface disorders. Ophthalmology. (1989) 96:709-22. doi: 10.1016/S0161-6420(89)32833-8

9. Holland EJ. Management of limbal stem cell deficiency : a historical perspective, past, present, and future. Cornea. (2015) 34:9-15. doi: 10.1097/ICO.0000000000000534

10. Burman S, Sangwan V. Cultivated limbal stem cell transplantation for ocular surface reconstruction. Clin Ophthalmol. (2008) 2:489-502.

11. Pellegrini G, Traverso CE, Franzi AT, Zingirian M, Cancedda R, De Luca M. Long-term restoration of damaged corneal surfaces with autologous cultivated corneal epithelium. Lancet. (1997) 349:990-3. doi: 10.1016/S0140-6736(96)11188-0

12. Man RC, Yong TK, Hwei NM, Wan Abdul Halim WH, Zahidin AZM, Ramli $\mathrm{R}$, et al. Corneal regeneration by induced human buccal mucosa cultivated on an amniotic membrane following alkaline injury. Mol Vis. (2017) 23:810-22.

13. Burillon C, Huot L, Justin V, Nataf S, Chapuis F, Decullier E, et al. Cultured autologous oral mucosal epithelial cell sheet (CAOMECS) transplantation for the treatment of corneal limbal epithelial stem cell deficiency. Investig Opthalmology Vis Sci. (2012) 53:1325. doi: 10.1167/iovs.11-7744

14. Kocaba V, Thépot A, Yamato M, Daisuke M, Kellal M, et al. Long-term results of cultured autologous oral mucosa epithelial cell-sheet (CAOMECS) graft for the treatment of blindness due to bilateral limbal stem cell deficiency. J Stem Cell Res Ther. (2014) 4:181. doi: 10.4172/2157-7633.1000181 keratinization during a prolonged time span. However, a study comparing allogenic CLET and COMEC suggested that oral mucosal epithelial cells have lower success, due to the higher incidence of post-operative PED and graft failure, and lower cell proliferation and differentiation activities. Allogenic LESCs may have a better ability to form a stable and integrated corneal epithelium.

\section{AUTHOR CONTRIBUTIONS}

OS and DG had the same contribution and should be considered first author. Database search was performed by DG and OS independently in order not to miss any relevant work in the researched field. The review was written and reviewed equally by both authors.
15. Wang J, Qi X, Dong Y, Cheng J, Zhai H, Zhou Q, et al. Comparison of the efficacy of different cell sources for transplantation in total limbal stem cell deficiency. Graefe's Arch Clin Exp Ophthalmol. (2019) 257:1253-63. doi: 10.1007/s00417-019-04316-z

16. Mariappan I, Kacham S, Purushotham J, Maddileti S, Siamwala J, Sangwan VS. Spatial distribution of niche and stem cells in ex vivo human limbal cultures. Stem Cells Transl Med. (2014) 3:1331-41. doi: 10.5966/sctm.2014-0120

17. Parihar JKS, Parihar AS, Jain VK, Kaushik J, Nath P. Allogenic cultivated limbal stem cell transplantation versus cadaveric keratolimbal allograft in ocular surface disorder: 1-year outcome. Int Ophthalmol. (2017) 37:1323-31. doi: 10.1007/s10792-016-0415-0

18. Daya SM, Watson A, Sharpe JR, Giledi O, Rowe A, Martin R, et al. Outcomes and DNA analysis of ex vivo expanded stem cell allograft for ocular surface reconstruction. Ophthalmology. (2005) 112:470-7. doi: 10.1016/j.ophtha.2004.09.023

19. Shimazaki J, Higa K, Morito F, Dogru M, Kawakita T, Satake Y, et al. Factors Influencing outcomes in cultivated limbal epithelial transplantation for chronic cicatricial ocular surface disorders. Am J Ophthalmol. (2007) 143:945-53. doi: 10.1016/j.ajo.2007.03.005

20. Kawashima M, Kawakita T, Satake Y, Higa K SJ. Phenotypic study after cultivated limbal epithelial transplantation for limbal stem cell deficiency. Arch Ophthalmol. (2007) 125:1337. doi: 10.1001/archopht.125.10.1337

21. Shortt AJ, Secker GA, Rajan MS, Meligonis G, Dart JK, Tuft SJ, et al. Ex vivo expansion and transplantation of limbal epithelial stem cells. Ophthalmology. (2008) 115:1989-97. doi: 10.1016/j.ophtha.2008.04.039

22. Pauklin M, Fuchsluger TA, Westekemper H, Steuhl K-P, Meller D. Midterm results of cultivated autologous and allogeneic limbal epithelial transplantation in limbal stem cell deficiency. Res Proj Dry Eye Syndr. (2010) 45:57-70. doi: 10.1159/000315020

23. Basu S, Fernandez MM, Das S, Gaddipati S, Vemuganti GK, Sangwan VS. Clinical outcomes of xeno-free allogeneic cultivated limbal epithelial transplantation for bilateral limbal stem cell deficiency. $\mathrm{Br} J$ Ophthalmol. (2012) 96:1504-9. doi: 10.1136/bjophthalmol-2012-301869

24. Prabhasawat P, Ekpo P, Uiprasertkul M, Chotikavanich S, Tesavibul N. Efficacy of cultivated corneal epithelial stem cells for ocular surface reconstruction. Clin Ophthalmol. (2012) 6:1483-92. doi: 10.2147/OPTH.S33951

25. Qi X, Xie L, Cheng J, Zhai H, Zhou Q. Characteristics of immune rejection after allogeneic cultivated limbal epithelial transplantation. Ophthalmology. (2013) 120:931-6. doi: 10.1016/j.ophtha.2012.11.001

26. Shortt AJ, Bunce C, Levis HJ, Blows P, Doré CJ, Vernon A, et al. Threeyear outcomes of cultured limbal epithelial allografts in aniridia and stevensjohnson syndrome evaluated using the clinical outcome assessment in surgical trials assessment tool. Stem Cells Transl Med. (2014) 3:265-75. doi: 10.5966/sctm.2013-0025

27. Zakaria N, Possemiers T, Dhubhghaill S, Leysen I, Rozema J, Koppen C, et al. Results of a phase I/II clinical trial: standardized, non-xenogenic, 
cultivated limbal stem cell transplantation. J Transl Med. (2014) 12:58. doi: 10.1186/1479-5876-12-58

28. Ramírez BE, Sánchez A, Herreras JM, Fernández I, García-Sancho J, NietoMiguel T, et al. Stem cell therapy for corneal epithelium regeneration following good manufacturing and clinical procedures. Biomed Res Int. (2015) 2015:410. doi: $10.1155 / 2015 / 408495$

29. Ganger A, Vanathi M, Mohanty S, Tandon R. Long-term outcomes of cultivated limbal epithelial transplantation: evaluation and comparison of results in children and adults. Biomed Res Int. (2015) 2015:480983. doi: $10.1155 / 2015 / 480983$

30. Chen P, Zhou Q, Wang J, Zhao X, Duan H, Wang Y, et al. Characterization of the corneal surface in limbal stem cell deficiency and after transplantation of cultured allogeneic limbal epithelial cells. Graefe's Arch Clin Exp Ophthalmol. (2016) 254:1765-77. doi: 10.1007/s00417-016-3410-2

31. Cheng J, Zhai H, Wang J, Duan H, Zhou Q. Long-term outcome of allogeneic cultivated limbal epithelial transplantation for symblepharon caused by severe ocular burns. BMC Ophthalmol. (2017) 17:8. doi: 10.1186/s12886-017-0403-9

32. Behaegel J, Zakaria N, Tassignon MJ, Leysen I, Bock F, Koppen C, et al. Shortand long-term results of xenogeneic-free cultivated autologous and allogeneic limbal epithelial stem cell transplantations. Cornea. (2019) 38:1543-9. doi: 10.1097/ICO.0000000000002153

33. Borderie VM, Ghoubay D, Georgeon C, Borderie M, de Sousa C, Legendre A, et al. Long-term results of cultured limbal stem cell versus limbal tissue transplantation in stage iii limbal deficiency. Stem Cells Transl Med. (2019) 8:1230-41. doi: 10.1002/sctm.19-0021

34. Campbell JDM, Ahmad S, Agrawal A, Bienek C, Atkinson A, Mcgowan NWA, et al. Allogeneic ex vivo expanded corneal epithelial stem cell transplantation: a randomized controlled clinical trial. Stem Cells Transl Med.(2019) 8:323-31. doi: $10.1002 /$ sctm.18-0140

35. Nishida K, Yamato M, Hayashida Y, Watanabe K, Yamamoto K, Adachi E, et al. Corneal reconstruction with tissue-engineered cell sheets composed of autologous oral mucosal epithelium. N Engl J Med. (2004) 351:1187-96. doi: 10.1056/NEJMoa040455

36. Chen H-CJ, Chen H-L, Lai J-Y, Chen C-C, Tsai Y-J, Kuo M-T, et al. Persistence of transplanted oral mucosal epithelial cells in human cornea. Investig Opthalmol Vis Sci. (2009) 50:4660. doi: 10.1167/iovs.09-3377

37. Nakamura T, Takeda K, Inatomi T, Sotozono C, Kinoshita S. Long-term results of autologous cultivated oral mucosal epithelial transplantation in the scar phase of severe ocular surface disorders. Br J Ophthalmol. (2011) 95:942-6. doi: 10.1136/bjo.2010.188714

38. Satake Y, Higa K, Tsubota K, Shimazaki J. Long-term Outcome of cultivated oral mucosal epithelial sheet transplantation in treatment of total limbal stem cell deficiency. Ophthalmology. (2011) 118:1524-30. doi: 10.1016/j.ophtha.2011.01.039

39. Priya CG, Arpitha P, Vaishali S, Prajna NV, Usha K, Sheetal K, et al. Adult human buccal epithelial stem cells: Identification, ex-vivo expansion, and transplantation for corneal surface reconstruction. Eye. (2011) 25:1641-9. doi: $10.1038 /$ eye. 2011.230

40. Sotozono C, Inatomi T, Nakamura T, Koizumi N, Yokoi N, Ueta M, et al. Visual Improvement after cultivated oral mucosal epithelial transplantation. Ophthalmology. (2013) 120:193-200. doi: 10.1016/j.ophtha.2012.07.053

41. Kolli S, Ahmad S, Mudhar HS, Meeny A, Lako M, Figueiredo FC. Successful application of ex vivo expanded human autologous oral mucosal epithelium for the treatment of total bilateral limbal stem cell deficiency. Stem Cells. (2014) 32:2135-46. doi: 10.1002/stem.1694
42. Dobrowolski D, Orzechowska-Wylegala B, Wowra B, Wroblewska-Czajka E, Grolik M, Szczubialka K, et al. Cultivated oral mucosa epithelium in ocular surface reconstruction in aniridia patients. Biomed Res Int. (2015) 2015:281870. doi: $10.1155 / 2015 / 281870$

43. Kim Y, Hyun JL, Jin SR, Kim YH, Jeon S, Choung HK, et al. Prospective clinical trial of corneal reconstruction with sheets. Cornea. (2017) 37:76-83. doi: 10.1097/ICO.0000000000001409.

44. Llames S, García-Pérez E, Meana Á, Larcher F, del Río M. Feeder layer cell actions and applications. Tissue Eng Part B Rev. (2015) 21:345-53. doi: 10.1089/ten.teb.2014.0547

45. de Araujo AL, Gomes JÁP. Corneal stem cells and tissue engineering: current advances and future perspectives. World J Stem Cells. (2015) 7:806-14. doi: 10.4252/wjsc.v7.i5.806

46. Hynds RE, Bonfanti P, Janes SM. Regenerating human epithelia with cultured stem cells: feeder cells, organoids and beyond. EMBO Mol Med. (2018) 10:139-50. doi: $10.15252 / \mathrm{emmm} .201708213$

47. Kim YJ, Lee HJ, Ryu JS, Kim YH, Jeon S, Oh JY, et al. Prospective clinical trial of corneal reconstruction with biomaterial-free cultured oral mucosal epithelial cell sheets. Cornea. (2018) 37:76-83. doi: 10.1097/ICO.0000000000001409

48. Li W, Hayashida Y, He H, Kuo C-L, Tseng SCG. The fate of limbal epithelial progenitor cells during explant culture on intact amniotic membrane. Invest Ophthalmol Vis Sci. (2007) 48:605-13. doi: 10.1167/iovs.06-0514

49. Mishan MA, Yaseri M, Baradaran-Rafii A, Kanavi MR. Systematic review and meta-analysis investigating autograft versus allograft cultivated limbal epithelial transplantation in limbal stem cell deficiency. Int Ophthalmol. (2019) 39:2685-96. doi: 10.1007/s10792-019-01092-x

50. Bains KK, Fukuoka H, Hammond GM, Sotozono C, Quantock AJ. Recovering vision in corneal epithelial stem cell deficient eyes. Contact Lens Anterior Eye. (2019) 42:350-8. doi: 10.1016/j.clae.2019.04.006

51. Ramachandran C, Basu S, Sangwan VS, Balasubramanian D. Concise review: the coming of age of stem cell treatment for corneal surface damage. Stem Cells Transl Med. (2014) 3:1160-8. doi: 10.5966/sctm. 2014-0064

52. Calonge M, Pérez I, Galindo S, Nieto-Miguel T, López-Paniagua M, Fernández I, et al. A proof-of-concept clinical trial using mesenchymal stem cells for the treatment of corneal epithelial stem cell deficiency. Transl Res. (2019) 206:18-40. doi: 10.1016/j.trsl.2018.11.003

53. Chakrabarty K, Shetty R, Ghosh A. Corneal cell therapy: with iPSCs, it is no more a far-sight. Stem Cell Res Ther. (2018) 9:1-15. doi: $10.1186 /$ s13287-018-1036-5

54. Cyranoski D. Woman is first to receive cornea made from reprogrammed stem cells. Nature. (2019) 573:482-5. doi: 10.1038/d41586-01902597-2

Conflict of Interest: The authors declare that the research was conducted in the absence of any commercial or financial relationships that could be construed as a potential conflict of interest.

Copyright (c) 2020 Samoila and Gocan. This is an open-access article distributed under the terms of the Creative Commons Attribution License (CC BY). The use, distribution or reproduction in other forums is permitted, provided the original author(s) and the copyright owner(s) are credited and that the original publication in this journal is cited, in accordance with accepted academic practice. No use, distribution or reproduction is permitted which does not comply with these terms. 